\title{
LE BASSIN LÉMANIQUE GALLO-ROMAIN
}

\author{
Michel TARPIN, Sylvie Berti, Marc-André Haldimann, \\ Frédéric Rossi, Lucie STEINER
}

\begin{abstract}
Mots-clés. Léman, Genève, Nyon, Lausanne, Sapaudia, port, ponts romains.
Key-words. Lake Leman, Geneva, Nyon, Lausanne, Sapaudia, harbour, Roman bridges.

Résumé. Malgré la dispersion des recherches archéologiques sur le lac Léman et son bassin, il est possible de dresser un bref bilan de ces recherches. Cette synthèse permet de montrer la continuité d'occupation de nombreux sites d'origine protohistorique. Inversement, on constate la disparition de la colonie de Nyon, créée ex nihilo par une décision politique. Nous supposons donc que les sites qui se développent vers la seconde moitié du II' s. avant J.-C. ont été établis en des lieux adaptés aux conditions climatiques locales, et en particulier aux variations du niveau du lac, et placés de manière à profiter au mieux d'un réseau de circulation terrestre et lacustre qui se développe dans le même temps et perdure à travers toute l'Antiquité.
\end{abstract}

\begin{abstract}
Although archaeological researchs on lake Leman are dispersed, it is possible to draw up an assesment of these researches. This final analysis shows that many sites of protohistoric origin had a permanent occupation. In contrast, the colony of Nyon, founded ex nihilo by political decision, disappeared. We can assume that the settlements which became important around the second half of the $2^{\text {nd }}$ century $B C$ were established on places adapted to local climatic conditions, and esperially to changes of the lake level; they were probably situated so that they could have benefit from the road and water transport network which increases in the same time and lasts through all the Antiquity.
\end{abstract}

Le plus grand lac d'Europe ${ }^{9}$ est au cœur d'un vaste bassin, clos en aval et en amont par des cluses, mais largement ouvert à la circulation intérieure. Rivières, lac et montagnes y définissent des paysages variés et une diversité climatique qui ont conditionné l'implantation humaine. Cette relation entre l'homme et le paysage n'est pas toujours immédiatement lisible. Il faut, pour l'identifier, confronter des constats d'ordre géographique, de rares sources écrites et des résultats archéologiques épars. Nous avons donc tenté de donner dans un volume limité une présentation méthodique d'une

9. Alimenté par un bassin de $6800 \mathrm{~km}^{2}$ (Guichonnet, 1994) ou $7945 \mathrm{~km}^{2}$ (Eschbach, 1990), le Léman, avec ses 89 millions de mètres cube d'eau, pour environ $580 \mathrm{~km}^{2}$, qui s'écoulent au rythme moyen de $240 \mathrm{~m}^{3} / \mathrm{s}$, est un des plus vastes réservoirs d'eau douce du monde. région dont l'unité est masquée par les divisions administratives. De ce fait, le bilan archéologique présente d'importantes disparités locales. C'est à travers cette documentation morcelée que nous tenterons de présenter le cadre naturel et humain durant l'Antiquité.

\section{LE LÉMAN DANS LE PAYSAGE ${ }^{10}$}

Le Léman antique n'avait pas la stabilité artificielle qu'on lui connaît aujourd'hui : P. Corboud estime à plus

10. Nous empruntons l'essentiel de notre description physique à Gabus et al., 1987; Eschbach, 1990 ; Guichonnet, 1994; Corboud, 1997 ; Wildi, 1997. 
de $7 \mathrm{~m}$ la variation du niveau du lac entre environ 4000 avant J.-C. (368 m, voire $366 \mathrm{~m}$, cf. Olive, 1972) et l'époque romaine, avec une malencontreuse lacune pour le Premier Âge du Fer. En basses eaux, il est presque possible de traverser la rade de Genève à pied sec, en période de hautes eaux, le courant est violent et rend la traversée difficile. Les niveaux de berges romains ont été identifiés à Genève (plus de $374,6 \mathrm{~m}$ à la fin du II $^{\mathrm{e}} \mathrm{s}$. avant J.-C. : pied du quai à $373,60 \mathrm{~m}$; autour de $376 \mathrm{~m}$ pour l'époque augustéenne) et à Lausanne-Vidy (estimé à $374,5 \mathrm{~m}$ au I ${ }^{\text {er }}$ s. après J.-C.) ; le niveau actuel est d'environ $372 \mathrm{~m}$. On a parfois expliqué certaines phases de haut niveau par des accidents, créant un barrage temporaire (Corboud, 1997), mais ce n'est pas démontré : les variations climatiques ont dû jouer un rôle déterminant. $\mathrm{Du}$ fait du régime torrentiel des affluents, qui contribuent à l'extension des grèves par alluvions, le lac connaissait en outre des variations saisonnières pouvant atteindre $2 \mathrm{~m}$ d'amplitude, découvrant et noyant alternativement les grèves et créant des lagunes. Pour la même raison, il arrive que le débit de l'Arve devienne aussi important que celui du Rhône à l'émissaire du lac. On ne peut donc pas appliquer au Léman les constats faits sur le Rhône en aval de Genève.

Depuis qu'il est observé, le climat lémanique s'avère très nuancé. $\mathrm{Si}$ le lac joue un rôle de régulateur (il y a plus de 2 degrés d'écart entre la côte et l'arrière-pays), on constate des différences de pluviosité marquées, avec un maximum vers Montreux et un minimum entre Genève et Thonon-les-Bains. Mais la région de Montreux est aussi plus chaude que la rive savoyarde. Plus de deux siècles d'observations montrent que les variations internes à la cuvette lémanique sont persistantes. On est donc en droit de supposer le même type de variabilité dans l'Antiquité. Cette diversité climatique génère des vents locaux, qui rythment la navigation sur le lac et soulèvent à certains endroits des vagues violentes.

La diversité des paysages de rives est une des caractéristiques de la région. Escarpées dans le haut lac, elles sont vallonnées sur le côté nord du grand lac et autour du petit lac. Par endroits, la pente rend les rives instables, comme en attestent plusieurs glissements de terrains récents. La tradition nous a conservé un écho d'une catastrophe de ce type en 563. D'après le récit de Marius, évêque d'Avenches ( $\mathrm{VI}^{\mathrm{e}} \mathrm{s}$.), il s'agit sans doute d'un tremblement de terre, qui aurait provoqué l'effondrement du Tauredunum - probablement l'actuel Grammont, à l'est de Villeneuve - et un raz de marée, qui détruisit des villages et même le pont de Genève ${ }^{11}$. Les nuances de relief ont, enfin, un impact sur la faune lacustre prélevable, puisque la «beine », cette zone presque plate qui s'étend à faible profondeur au-delà de la grève inondée, et qui abrite une vie intense, est pratiquement inexistante dans certains secteurs du haut lac. La rive du Léman est donc un milieu attirant, du fait de son climat, de l'irrigation naturelle des terres, des possibilités de pêche et de la commodité des déplacements, mais aussi instable, sujette à des inondations et exposée aux vagues.

S'il nous faut imaginer le paysage lémanique à partir de données modernes, c'est que les sources antiques sont avares de données. Le cours du Rhône et le Léman sont connus dès l'époque hellénistique ( $c f$. Apollonios de Rhodes, Argonautiques, 4, 619-650), mais l'imprécision paraît être la règle. Strabon $(4,3,3)$ pensait encore que le Rhin et le Rhône communiquaient, et plaçait les Nantuates du Chablais sur le haut Rhin. Il dit seulement du Léman $(4,1,11)$ que c'est un grand lac, et que le cours du Rhône reste visible lorsqu'il se jette dans le lac. Aucune source n'évoque la navigabilité du lac ou du fleuve, ni ne parle de la pêche ou de produits locaux importés à Rome. On peut cependant déduire des tentatives de traversée du Rhône par les Helvètes que le Rhône était navigable - et dépourvu de gué - entre Genève et le Fort de l'Écluse (Flutsch, 1992). Le Léman est connu de César (La guerre des Gaules, 1, 8, 1), Strabon $(4,1,11)$, Pomponius Mela $(2,74 ; 79)$, Pline l'Ancien (Histoire naturelle, 3, 4, 33), Dion Cassius $(39,5,2)$ et Avienus (Ora maritima, 674 sq.) sous le nom de Lemannus ou Lemmanus. Il prend tardivement le nom de « lac de Lausanne ", attesté sur la Table de Peutinger et dans l'Itinéraire d'Antonin.

\section{LA PÉNÉTRATION ROMAINE : $\mathrm{II}^{\mathrm{e}}-\mathrm{I}^{\mathrm{er}}$ S. AVANT J.-C.}

Pendant près d'un siècle, de 120 environ à 58 avant J.-C., le Léman a fait figure de trait d'union entre la

11. Cf. Favrod, 1991, p. 78-81. Voir aussi Grégoire de Tours, Histoire des Francs, IV, 31, trad. R. Latouche, Paris, 1996, p. 213-214 ; Favrod, 1991, p. 102-103. Ce récit ne figure dans aucune autre source et n'a encore jamais été confirmé par une étude géologique. 


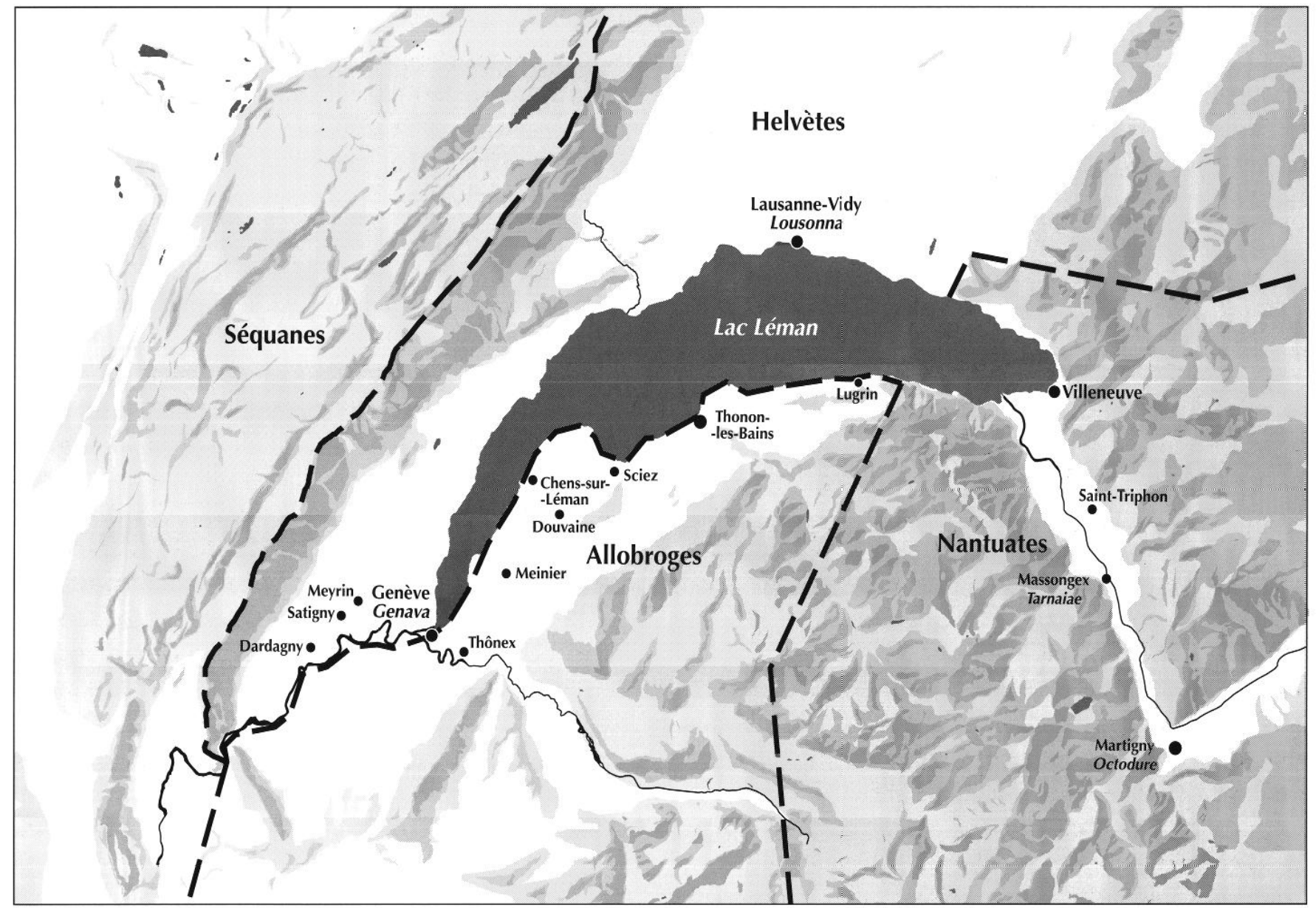

Fig. 11 - Principaux sites de La Tène cités dans le texte (dessin Archéodunum, SA).

province de Transalpine et la Gaule indépendante. D'ailleurs, aucun élément de la vie courante ne traduit une différence entre Helvètes et Allobroges. Seuls les Nantuates du Chablais se démarquent par l'emploi massif de céramiques culinaires non tournées à paroi fine et à dégraissant de talcschiste, qui signent leur appartenance à la sphère alpine (Curdy, in : Haldimann et al., 1991, p. 146-147). La documentation, pour cette période, est cependant peu homogène, et concentrée en grande partie à Genève (fig. 11). En outre, l'occupation du territoire dans son ensemble ne peut être discutée de façon pertinente, car la demi-douzaine de sites ruraux connus, attestés seulement par une poignée de tessons, se trouvent dans le canton de Genève, et les quelques sépultures mises au jour autour de Genève, de Vidy, à Vevey et dans le Chablais ne sont pas datées avec précision (Martin-Kilcher, 1981; Kaenel, 1990, p. 322-323). Le rôle commercial du lac paraît cependant important.

En effet, à Genève, la berge méridionale, au pied de la colline Saint-Pierre, fréquentée dès le milieu du $\mathrm{II}^{\mathrm{e}} \mathrm{s}$. avant J.-C., accueille un bassin portuaire en oméga, réalisé en pieux de chêne, abattus en 122-121 avant J.-C. (Bonnet et al., 1989) (fig. 12). Cet aménagement se poursuit en aval du port entre 112 et 77 avant J.C. : une digue supportant sans doute une voie a été repérée (Bonnet, 1992a, p. 9-11). Historiquement attesté, le pont enjambant le Rhône en tirant partie de l'île n'est en revanche pas daté précisément, mais il peut difficilement être postérieur aux autres ponts de la région. Deux d'entre eux ont pu être datés : le premier permettait le franchissement de l'Arve à Carouge (datation dendrochronologique : 100 avant J.-C.) et le second de la Laire entre Onex et Plan-lesOuates (datation ${ }^{14} \mathrm{C}$ : entre 135 et 75 avant J.-C.) 


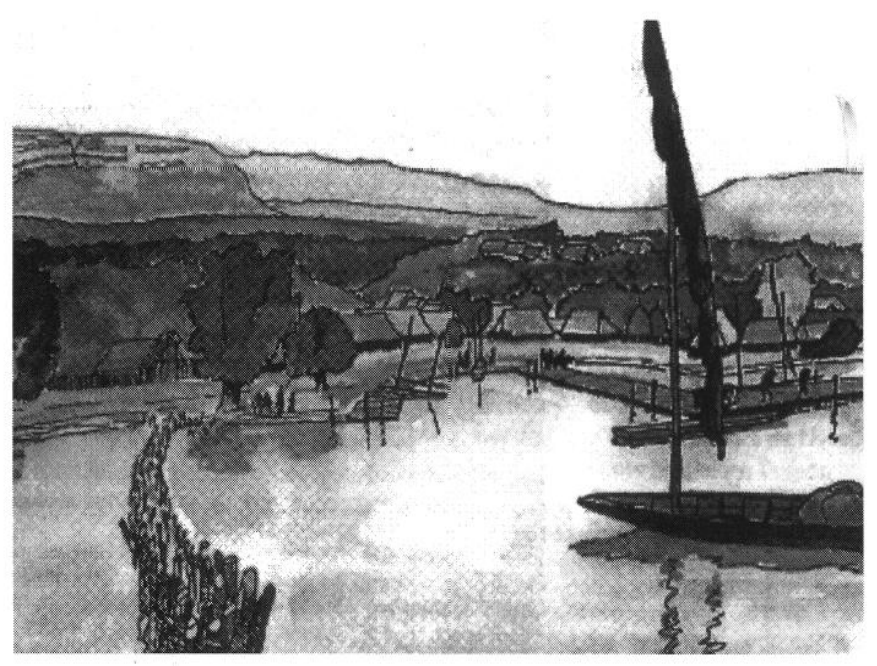

Fig. 12 - Reconstitution graphique de la rade de Genève vers 120 avant J.-C. (dessin G. Deuber, service cantonal d'archéologie, Genève).

(Bonnet, 1992b, p. 15 ; 1994, p. 51-52). Ils témoignent de l'élaboration cohérente d'un réseau routier complet. Sur la rive droite, la voie prolongeant le pont vers la colline de Saint-Gervais est établie avant le milieu du $\mathrm{I}^{\mathrm{er}} \mathrm{s}$. avant J.-C. ; elle est bordée par un temenos qui abrite plusieurs menhirs (Bonnet, Privati, 1991, p. 205). Dans l'ensemble, Genève couvre près de 20 ha entre la rive du lac et la colline de Saint-Pierre, peut-être fortifiée par un réseau de fossés, définissant un éperon barré (Paunier, 1981, p. 5051 ; Haldimann et al., 1991, p. 194-195 ; Bonnet, 1992a, p. 9-12). Au point culminant de la colline, un fossé délimite une zone privilégiée, dévolue soit à une citadelle (arx), soit à une aire cultuelle (Blondel, 1941, p. 102-105 ; Bonnet, 1984, p. 46).

En outre, plusieurs établissements ruraux, de plan et d'importance inconnus, ont livré du mobilier caractéristique de La Tène D : Meinier (Bonnet, 1986, p. 62), Dardagny-Pont de Brive (Paunier, 1981, p. 133), Meyrinle Cern (Paunier, 1981, p. 150), Satigny-en Mornex (Paunier, 1981, p. 150-152), Thônex (Terrier et al., 1994, p. 104-105). S'y sont ajoutés récemment deux sites : Vandœuvres et le Parc de La Grange (Bonnet, 1994, p. 42), qui livrent une céramique caractéristique de la fin du $\mathrm{II}^{\mathrm{e}}$ ou de la première moitié du ${ }^{\mathrm{er}} \mathrm{s}$. avant J.-C. On remarquera la répartition de ces sites sur les deux rives du lac (fig. 11) : la présence d'une éventuelle frontière entre le territoire allobroge, intégré depuis 122-121 avant $\mathrm{J}$-C., et le territoire helvète ne paraît donc pas avoir d'ef- fet sur l'habitat. En 58 avant J.-C., César rencontre ainsi une campagne déjà modelée par un réseau routier élaboré et jalonnée par des établissements conséquents. En revanche, les traces de l'histoire événementielle demeurent indécelables sur le terrain. Aucune fortification de la rive gauche du Rhône ni aucune destruction en milieu urbain n'ont été repérées à ce jour (Flutsch, 1992 ; Haldimann et al., 1997).

Lausanne paraît jouer alors un rôle équivalent à celui de Genève. Des vestiges d'habitat, datés entre 100 et 50 avant J.-C., ont été identifiés sur la colline de la cathédrale de Lausanne (Egloff, Farjon, 1983, p. 29-30). En outre, la présence à Vidy, sous le vicus du Haut-Empire, de trente tombes attribuées à La Tène D1 (Kaenel, 1995, p. 70-71), remet en cause l'hypothèse d'une fondation $e x$ nihilo de l'agglomération gallo-romaine. La continuité d'occupation du site semble d'ailleurs attestée par une fosse, datée par ${ }^{14} \mathrm{C}$ entre 387 et 129 avant J.-C., dans un lieu de culte signalé par deux menhirs, qui accueillera un enclos rituel de tradition celtique, puis un fanum dès l'époque flavienne (Paunier et al., 1989). Il y a donc peutêtre eu deux habitats contemporains formant une seule entité, l'un sur la colline de la cathédrale et l'autre (un port ?) sur la berge du lac, au débouché du Flon (Haldimann et al., 1997).

En amont, seule Tarnaiae-Massongex, alors capitale des Nantuates et siège du sanctuaire fédéral des quatre peuples occupant la haute vallée du Rhône (Nantuates, Véragres, Sédunes et Ubères), probable point de rupture de charge et premier pont routier en amont du Léman, a livré des traces ténues d'une fréquentation des berges du Rhône dès la fin du II ${ }^{e}$ s. avant J.-C. Il faut y ajouter le site de Saint-Triphon, peu éloigné des rives du Rhône (Kaenel et al., 1984). Au sud, le peuplement de la rive française, connu seulement par des observations anciennes, parâil clairsemé ; seul un oppidum est signalé à Champanges, à proximité de Thonon-les-Bains. Une nécropole a pu être observée à Chens-sur-Léman et une tombe isolée à Douvaine. En outre, quelques trouvailles monétaires signalent une fréquentation des lieux à Sciez, Thonon-les-Bains et Lugrin (Willigens, 1991). Mais la carence des données archéologiques pour cette période est soulignée par le port de Genève. Cet aménagement important suppose forcément la présence d'autres installations contemporaines, qui n'ont pas encore été identifiées, sur les rives et l'existence d'un trafic assez soutenu sur le lac. En outre, la découverte de deux statues en bois 


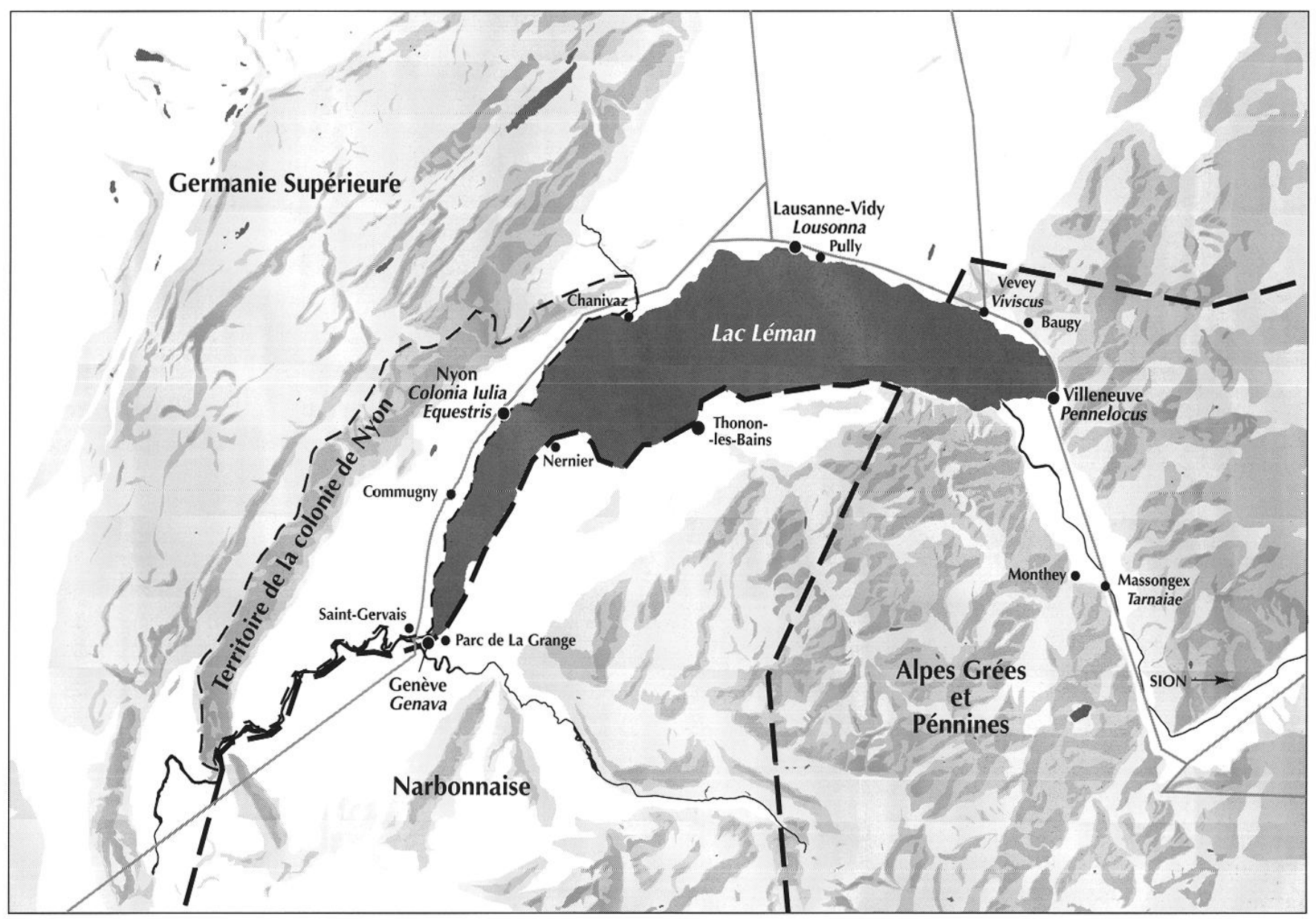

Fig. 13 - Principaux siles gallo-romains cités dans le lexte (dessin Archéodunum, SA).

à Genève (datée autour de 80 avant J.-C.) et à Villeneuve (atribuée à La Tène D) semble attester la présence de cultes sur les berges mêmes du lac (Wyss, 1979 ; Bonnet et al., 1989).

\section{ROME SUR LE LÉMAN : LE HAUT-EMPIRE}

Trait d'union entre le col du Grand Saint-Bernard, définitivement contrôlé après les campagnes de 16-14 avant J.-C., voire celle de 25 avant J.-C., et le bassin rhénan, de même que prolongement du couloir rhodanien en direction des Alpes et du Rhin, le Léman a vu s'établir sur ses rives de nombreux sites gallo-romains (fig. 13), dans le même temps qu'était développé un important réseau rou- tier. D. Van Berchem ${ }^{12}$ suppose ainsi que la route rapide, mais difficile, de la rive gauche a été supplantée par une route, plus longue mais plus confortable, en rive droite, passant par Lausanne et Nyon. Il était ainsi possible de rejoindre la Saône par Lausanne, Orbe et Besançon, ou le Rhin depuis Vevey, par Yverdon (voie d'eau) ou Moudon, Avenches, Soleure et Augst (voie terrestre). De fait, la Table de Peutinger connait seulement l'itinéraire de la rive droite. En aval de Genève, on restitue une route principale en rive gauche du Rhône, de Genève à Seyssel, évitant ainsi le passage délicat du Fortde-l'Écluse (Dufournet, 1995). La navigation sur le lac est

12. Cf. Van Berchem, 1987. C'est très certainement par la rive gauche qu'est passé Caalba après sa défaite à Octodure (César, La guerre des Gaules, 3, 3, 6, 5). 


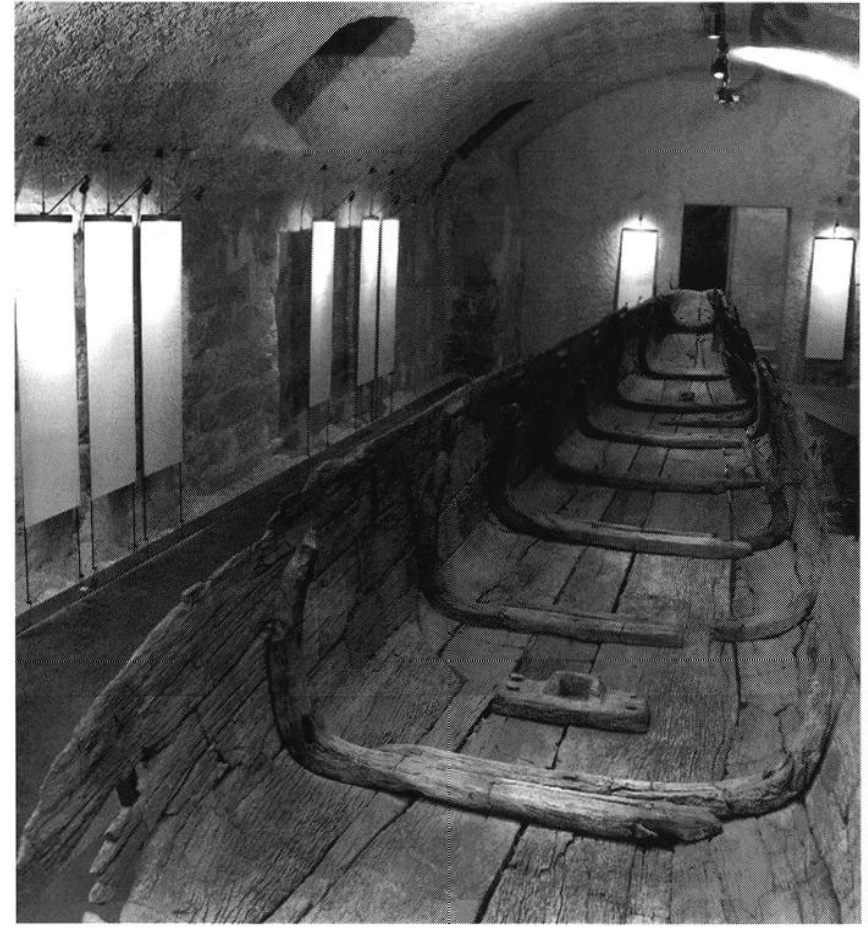

Fig. 14 - Barque de la fin du $I V^{*}$ s., exposée au musée d'Yverdonles-Bains (photo Y. André, Société du musée et Vieil-Yverdon).

organisée au profit d'une corporation de nautes du Léman. L'archéologie n'a pas encore identifié d'épave de bateau lémanique antique, mais ils devaient être du type des barques romaines d'Yverdon (Weidmann, Klausener, 1985 ; Arnold, 1992 ; Terrier, 1997) (fig. 14). L'épigraphie mentionne en outre des ratiarii superiores, sans doute basés à Genève (CIL XII, 2597; H. M., 108; $R I S, 15)$, qui assuraient la navigation en amont de Lyon plutôt que seulement de Bellegarde ("perte du Rhône ») à Genève (Van Berchem, 1982, p. 107). Les nautes du Léman prenaient leur suite sur le lac, peut-être jusqu'à Massongcx (prcmicr pont en amont de Genève). L'unité géographique que constitue le bassin lémanique ne correspondait pourtant à aucune entité administrative. Genève et la côte méridionale appartenaient à la province de Narbonnaise, tandis que le territoire des Helvètes fut attribué à la Gaule Belgique avant d'être rattaché à la Germanie Supérieure vers 85-89. L'extrémité orientale du lac fut d'abord réunie à la Rhétie-Vindélicie vers 15 avant J.-C., puis dépendit, depuis le règne de Claude, de la province des Alpes Pénnines.

Malgré ces divisions politiques, on peut considérer que la colonie de Nyon fait office de «capitale » officieuse du Léman. C'est en effet la seule colonie de la région. En outre, elle seule possède l'ensemble des édifices publics et le cadre monumental propres à représenter l'image de Rome. Comme l'indique son nom, la colonia Iulia Equestris a été fondée par César ou un de ses lieutenants, probablement entre 50 et 44 avant J.-C., par déduction de soldats issus de la cavalerie légionnaire (Van Berchem, 1982, p. 47-53), sur un site que l'on suppose maintenant libre de toute occupation. Certes, au Bas-Empire, Nyon portait aussi le nom de Nouiodunum ${ }^{13}$. Mais cette appellation indigène s'appliquerait à Nyon, non en souvenir d'un établissement celtique mais plutôt par opposition à l'ancien bourg gaulois de Genaua, distant d'une vingtaine de kilomètres (Rossi, 1995, p. 102-110). Par ailleurs, une nouvelle interprétation du mobilier a montré qu'il n'y a pas de lacune entre la date de fondation historique et les premiers vestiges archéologiques, ténus mais bien réels (Rossi, 1995). Les vétérans sont absents de l'épigraphie locale, qui livre en revanche des noms de grandes familles viennoises. Sur la base de textes médiévaux, dont les plus anciens remontent aux $\mathrm{X}^{\mathrm{e}}$-XII ${ }^{\mathrm{c}}$ s. (Duparc, 1979, p. 363-370), et qui citent un pagus Equestricus, on restitue un territoire qui s'étendait sans doute de la rive droite du Rhône jusqu'à l'Aubonne, et du Jura aux rives du Léman, aux confins des Helvètes, des Séquanes et des Allobroges (fig. 13). La répartition des bornes milliaires comptant les distances à partir de Nyon correspond à peu près à cette région, avec un léger débordement en direction de Lausanne-Vidy (Howald, Meyer, 1940).

$\mathrm{Au}$ contraire de Genève, Nyon a connu, dès les dernières années avant notre ère, un urbanisme programmé et régulier. Pendant le règne de Tibère (1437), la ville connaît une importante phase de monumentalisation progressive : vers le milieu du ${ }^{\mathrm{er}}$ s., le centre monumental occupe pratiquement huit insulae. Dernier équipement majeur de la colonie, l'amphithéâtre, érigé au début du $\mathrm{II}^{\mathrm{e}}$ s., prend place en dehors du tissu urbain (fig. 15). Faute de vestiges d'enceinte, le périmètre de l'agglomération ne peut être défini qu'au regard des trouvailles funéraires, en particulier de deux nécropoles, l'une le long de la route menant à Lausanne, l'autre en direction de Genève. Les collines et plateaux proches de la ville ont

13. Il est attesté par la Notitia Galliarum (rédigée entre 386 et 450 ), qui cite " ciuitas Equestris id est Nouiodunum "; Monumenta Germaniae Historica, Auctores antiquissimi, 9, 1, Chronica Minora, 1 (Th. Mommsen), p. 256. 
accueilli des quartiers à vocation commerciale et de riches villae suburbaines, connus de manière partielle. Entre l'amphithéâtre et le lac, des bâtiments ont été repérés, ainsi que des traces de conduites en bois et un puits aménagé au moyen d'un tonneau (construit en sapin blanc abattu vers 23 après J.-C.). Il est tentant de mettre ces aménagements en relation avec le port antique de Nyon, qui n'a pas encore été retrouvé, mais qui se trouvait probablement au même emplacement que le port du Moyen Âge, situé à proximité immédiate sur le plan de 1672.

À en juger par des fouilles pourtant importantes, les espaces dévolus à l'habitat privé semblent limités au regard du cadre monumental (seules quatre grandes domus - entre 900 et $1200 \mathrm{~m}^{2}$ - ont livré un plan intelligible). On en vient à se demander si Nyon, située entre les deux anciennes agglomérations de Genève et Lausanne, n'aurait pas joué surtout le rôle de villevitrine, marquant la présence de Rome dans la région lémanique. Sa position lui permettait certes de contrôler d'éventuelles migrations vers le sud, mais c'est aussi un site enfermé entre Jura et lac, au contraire des vieilles villes de Genève, Lausanne (et Vevey ?), mieux placées pour contrôler le trafic commercial.

Sous l'Empire, Genève voit son importance s'accroître. La ville est alors un vicus de la cité de Vienne, soit un relais de l'autorité administrative, fort éloignée. Malgré plus d'un siècle d'archéologie, le plan d'ensemble de Genava (ou Genua) demeure lacunaire. C'est néanmoins l'agglomération la plus importante du bassin lémanique : près de 35 ha sur la rive gauche, et 10 ha sur la rive droite dans le courant $d u \mathrm{II}^{\mathrm{c}} \mathrm{s}$. de notre ère. Genève n'a pas connu de plan régulateur orthogonal : seuls les deux axes principaux se croisent à angle presque droit, la voirie secondaire se pliant aux fortes contraintes topographiques. Le caractère peu homogène du tissu urbain est d'ailleurs démontrè (Haldimann, Ramjoué et al., 1991, p. 195-202). L'axe principal est-ouest conserve le tracé protohistorique : il aboutit au pont sur le Rhône, puis remonte la colline de Saint-Gervais avant de quitter la ville pour rejoindre la voie romaine du pied du Jura (Blondel, 1933; Bonnet, 1996, p. 26-28, 35). La rive gauche paraît accueillir l'essentiel des activités commerciales, comme en témoigne la construction d'un nouveau port à l'emplacement de celui de la seconde moitié du $\mathrm{II}^{\mathrm{e}} \mathrm{s}$. avant J.-C. Les rangées de pilotis (datation dendrochronologique : entre 28 et 47 après J.-C.) mis en œuvre pour servir de contreforts à deux digues, ainsi qu'un bas-

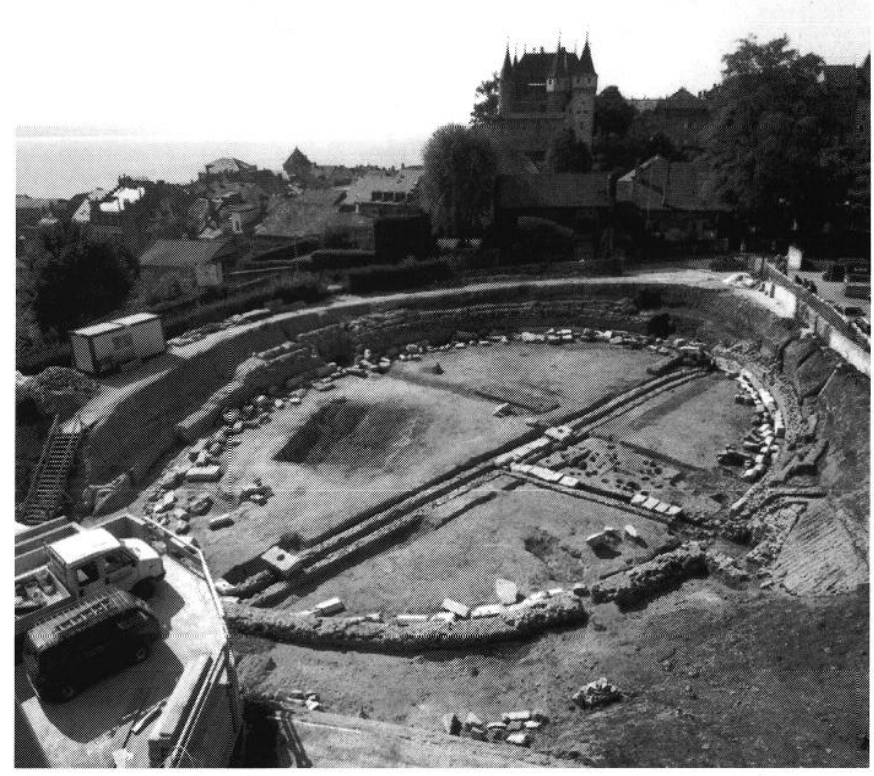

Fig. 15 - Amphithéâtre de Nyon datant du début du II' s. après J.-C. (photo Fibbi-Aeppli Crrandson).

sin aboutissant aux fondations d'un bâtiment permettent la reconquête d'une berge jusqu'alors fluctuante. En effet, la digue augustéenne a été observée environ $2 \mathrm{~m}$ au-dessus des installations du $\mathrm{II}^{\mathrm{e}} \mathrm{s}$. avant J.-C. La présence d'entrepôts et de thermes le long des rives du lac est attestée par les fouilles anciennes (Paunier, 1981, p. 77-88; Bonnet et al., 1989, p. 6-7). Sur la rive droite, ne sont actuellement bien identifiés que les vestiges d'un sanctuaire dont l'origine remonte au milieu du $\mathrm{I}^{\mathrm{er}} \mathrm{s}$. avant J.-C. Remanié plusieurs fois (Bonnet, Privati, 1991, p. 205-208), il ne disparaitra que dans le courant du IV ${ }^{\mathrm{e}}$. au profit d'une vaste église funéraire.

Le vicus Genavensis semble florissant jusqu'à la fin du $\mathrm{II}^{\mathrm{e}}$ s. Un incendie violent, daté par des monnaies dont la plus récente est frappée en $175 / 176$, détruit l'insula élevée à l'emplacement de la domus tibérienne du plateau des Tranchées (Haldimann, Ramjoué et al., 1991, p. 199). Cet emplacement, situé au cœur de l'agglomération antique, demeure dès lors abandonné, ce qui soulève quelques interrogations sur l'état réel de Genève pendant la première moitié du III ${ }^{e}$ s., période pourtant prospère sur les rives du Léman.

Tout comme Genève représente la ville la plus septentrionale de Narbonnaise, le vicus de Lausanne-Vidy est l'agglomération la plus occidentale de la cité des Helvètes. La ville, qui est organisée en longueur le long de la voie 
menant à Nyon, connaît un important développement urbain dès les années 40-20 avant J.-C. (Pichard Sardet $e t$ al., 1993). Outre de nombreuses maisons particulières, plusieurs bâtiments publics ont été dégagés. Une importante activité artisanale y régnait, notamment dans le domaine de la métallurgie et de la poterie. La présence d'inscriptions des nautes du Léman trahit la vocation commerciale de Lausanne, à un point de rupture de charge et à la convergence de plusieurs voies terrestres. Il est fort probable que ces nautes avaient leur siège social à Vidy ; ils y possédaient en tous cas une schola près de la basilique ${ }^{14}$.

L'agglomération se situe entre les deltas du Flon et de la Chamberonne. Les aménagements côtiers de la fin du $\mathrm{I}^{\text {er }}$ s. sont établis sur une séquence sablo-graveleuse. Le "port » n'était donc sans doute qu'une plage située au fond d'une baie encadrée par les deux deltas. La rive du lac était stabilisée sur près de $1 \mathrm{~km}$ par un enrochement localement fondé sur des pieux de chêne (datation dendrochronologique : après 73 après J.-C.). Cette digue, repérée à l'avant des constructions du forum et à l'ouest de l'agglomération, à proximité des quartiers d'entrepôts, servait à lutter contre les effets des importantes variations saisonnières du niveau du lac et à briser les vagues de tempête. Ces enrochements retenaient en outrc les quais où l'on stockait lcs marchandiscs. Les fouilles ont permis d'identifier trois autres aménagements de rive. Un alignement de pieux (sommet à $373,50 \mathrm{~m}$ ) d'environ $20 \mathrm{~m}$ de longueur, découvert en 1990 , forme vraisemblablement une simple palissade aménagée pour lutter contre l'ensablement de la côte. Dans ce secteur du vicus, l'existence d'un bassin ou d'un chenal ménageant un accès au rivage protégé de l'ensablement paraît plausible, car on y a découvert en 1921 les vestiges d'un enrochement perpendiculaire à la rive du lac (digue de port ?) et d'un bâtiment dont le plan rappelle celui d'un entrepôt. Plus à l'est, à proximité de la basilique, les vestiges d'un perré ont été repérés en 1935-1940. Enfin, il faut mentionner un remblai, constitué de pierres et de tuiles entassées sur plusieurs centimètres, déposé sur le fond lacustre à l'avant de la digue de stabilisation de la côte. Tout en réduisant l'action des vagues, en empêchant le mouvement des sédiments, cet aménagement limitait le mouvement des sables. En effet, sa structure, comportant des vides importants entre les pierres, constituait un piège à sédiments. Par ailleurs, il

14. Cf. Collart, Van Berchem, Revue historique vaudoise, 1939, p. 127. représentait un moyen de faire face aux variations du niveau du lac : stable et en pente douce, il permettait un accès facile aux bateaux éloignés du bord lors des basses eaux. En effet, à Vidy, où la pente moyenne de la plage se situe autour de $3 \%$, une baisse des eaux de $1 \mathrm{~m}$ faisait reculer la ligne de rivage de $30 \mathrm{~m}$. Ainsi, les bateaux ne pouvaient accoster contre le perré, dont la base se situe aux alentours de $375 \mathrm{~m}$, qu'en période de hautes eaux. Le reste de l'année, les barques étaient simplement hissées sur le rivage. Lousonna était donc vraisemblablement un port d'échouage. En outre, Vidy se trouve sur une côte d'alluvions : les problèmes d'ensablement étaient donc aigus, mais les alluvions accumulées sous forme de plage fournissaient une mise à sec pratique et appréciée de tous temps pour des barques à fond plat. Si sommaires que soient les installations portuaires, leur extension sur près de $1 \mathrm{~km}$ de long suffit à montrer qu'il s'agissait d'un port actif, capable de gérer un trafic important.

Autre point important de la circulation lémanique, quoique appartenant à la sphère culturelle alpine, le site de Massongex doit être identifié avec Tarnaiae, capitale du peuple gaulois des Nantuates et sanctuaire de Taranis (Van Berchem, 1982, p. 172-184; Haldimann, Curdy et al., 1991 ; Wiblé, 1994). À cet endroit, situé en aval de la clusc de Saint-Mauricc, la voic du Grand Saint-Bcrnard traversait le Rhône par un pont pour rejoindre la rive droite en direction de Villeneuve-Pennelocus et VeveyViviscus. L'agglomération s'étend sur moins de 4 ha et abrite un vaste complexe thermal. Des entrepôts et des habitations bordent deux chaussées reliant le pont à la voie de la rive gauche. L'agglomération demeurera florissante durant tout le Haut-Empire.

D'autres sites lémaniques moins bien connus ont dû participer au trafic attesté à Genève, Lausanne ou Massongex. Ainsi, le site antique de Vevey-Viviscus, point de rupture de charge en direction du plateau suisse ( $c f$. supra), qui n'était connu que par des découvertes fortuites et des mentions dans les sources géographiques, est depuis peu partiellement fouillé (Paratte, 1997). L'habitat présente un faciès proche des maisons de Lausanne-Vidy. La présence d'un milliaire donnant la distance de Martigny incite à voir dans Vevey un site extrême des Alpes Pénnines. Villeneuve, où les vestiges archéologiques sont fort ténus, a été identifiée avec le site antique de Pennelocus (Viollier, 1927; Staehelin, 1948, p. 342). Ces vestiges n'autorisent pas d'interprétation du site. La position de Villeneuve, entre Vevey et 
Massongex, à l'extrémité est du lac Léman, en fait surtout une étape sur la route de Martigny à Lausanne, plutôt qu'un carrefour routier. Ce pourrait être cependant un point de rupture de charge, destiné à éviter le courant violent du Rhône à l'embouchure. Enfin, l'agglomération gallo-romaine de Thonon, dont le nom antique nous échappe, reste très mal connue. Un quartier artisanal, où se trouvait un atelier de potier tardif, a notamment fait l'objet d'une fouille récente. La présence d'un port est parfois déduite du toponyme "Puers" (Broise, 1974, p. 76). Du fait des lacunes de l'archéologie savoyarde, il faut peut-être supposer d'autres agglomérations sur la rive sud du Léman.

Peu de villae ont été fouillées sur les rives du Léman. Pourtant, les prospections aériennes donnent une densité d'environ une villa $/ \mathrm{km}^{2}$ pour le canton de Genève (Paunier, 1981, p. 282). Les plus spectaculaires, comme celles de Pully, de Commugny (Chatelain, 1976; Weidmann, 1978) ou de Baugy, sur la côte vaudoise, montrent un net penchant des propriétaires pour les sites élevés, proches des rives et dotés d'une vue étendue sur le lac et les Alpes. La mieux connue de ces villae est celle du Parc de la Grange (Bonnet, 1994, p. 41-47; 1996, p. 33-34). Établie dans une enceinte de $400 \mathrm{~m} \times 200 \mathrm{~m}$, en un lieu occupé à l'Âge du Bronze final, la pars urbana a livré un réseau de fossés et un dépotoir remontant à la fin du II ${ }^{e}$ ou au début du I ${ }^{\text {er }}$ s. avant J.-C. Leur comblement permettra l'édification de plusieurs bâtiments en bois, construits entre 80 et 20 avant J.-C. et remplacés à partir de 50 après J.-C. par une grande résidence à péristyle. Elle sera à plusieurs reprises agrandie jusqu'au BasEmpire et perdurera partiellement jusqu'au XII ${ }^{e}$ s. Les bâtiments qui composent la pars rustica sont entretenus pendant le $I^{e} \mathrm{~s}$. avant d'être reconstruits au $\mathrm{V}^{\mathrm{e}} \mathrm{ou}$ au $\mathrm{VI}^{\mathrm{e}} \mathrm{s}$. Un vaste édifice est érigé postérieurement au $\mathrm{V}^{\mathrm{e}} \mathrm{s}$. entre la berge du lac et la clôture septentrionale du domaine. Il s'agit probablement d'une nouvelle résidence, utilisée jusqu'au $\mathrm{VIII}^{\mathrm{e}}$ ou $\mathrm{IX}^{\mathrm{e}} \mathrm{s}$. Une digue et une tour médiévale oblitèrent en partie ce puissant bâtiment ; ces structures seront utilisées jusqu'au XIV ${ }^{\mathrm{e}} \mathrm{s}$. au moins.

Nous ne savons rien du fonctionnement économique des villae lémaniques, mais la présence d'un important établissement, repéré par photo aérienne et connu par des découvertes anciennes, à Chanivaz (Buchillon), exactement à l'embouchure de l'Aubonne, amène à poser la question d'une éventuelle pisciculture, selon un modèle bien connu dans les villae maritimes italiennes.

\section{LE LÉMAN CHRÉTIEN : L'ANTIQUITÉ TARDIVE}

La période qui va, en gros, de Dioclétien au VI ${ }^{\mathrm{e}} \mathrm{s}$. présente, au sein d'un cadre humain apparemment stable, de profondes mutations institutionnelles et culturelles. Ainsi, après les réformes de Dioclétien, la rive nord du Léman est intégrée dans une nouvelle province, la Maxima Sequanorum, qui a pour capitale Besançon et regroupe les cités de Nyon, Bâle et Avenches. Genève, promue au rang de cité probablement dans le dernier tiers du III ${ }^{\mathrm{e}}$ s., et dont le territoire englobe la rive sud du Léman, appartient à la Viennoise, alors que l'autre extrémité du lac revient à la cité du Valais, dans les Alpes Grées et Pénnines (Van Berchem, 1982, p. 253-263 ; Fellmann, 1992, p. 313) (fig. 16). D'autre part, la christianisation est bien attestée dès le $I^{\mathrm{e}} \mathrm{s}$. Le premier évêque de Genève, Isaac, est connu vers 400 , alors que celui du Valais, Théodore (ou parfois Théodule), qui réside dans un premier temps à Martigny, signe les actes du concile d'Aquilée en 381 (Vischer, 1995, p. 17-34). Martigny est dotée d'une cathédrale dès la fin du IVe s. (Lehner et al., 1993). Mais la cité des Helvètes ne reçut pas d'évêque avant le début du $\mathrm{VI}^{\mathrm{e}} \mathrm{s}$. Le siège s'établit alors en alternance à Avenches et Vindonissa, avant de se fixer à Lausanne, vers la fin de ce siècle (Chevalley, Favrod, 1992). Le développement du groupe épiscopal de Genève, ainsi que des églises funéraires de La Madeleine et de Saint-Gervais, marquent très tôt l'empreinte de la christianisation dans la ville (Bonnet, 1977 ; 1993 ; Bonnet, Privati, 1995). Selon D. Chevalley et $\mathrm{J}$. Favrod, l'évêché de Genève, au $\mathrm{V}^{\mathrm{e}} \mathrm{s}$., aurait englobé non seulement la ciuitas Equestrium, mais aussi la cité des Helvètes. La réunion de ces trois cités aurait également formé une nouvelle circonscription administrative, couvrant le Léman, la Sapaudia, dans laquelle le général romain Aetius installa les Burgondes en 443 après J.-C. (Chevalley, Favrod, 1992, p. 65-67; Favrod, 1997, p. 100-117). Genève serait ainsi logiquement devenue la première capitale de leur royaume, avant de partager ce statut avec Lyon. Cette installation des Burgondes, qui constitue un fait historique marquant, présente un impact archéologique limité, car il est en effet très difficile d'isoler des groupes de sépultures que l'on puisse mettre en relation avec l'installation de ce peuple (Gaillard de Sémainville éd., 1995 ; Martin, 1995). Mais les mobiliers spécifiques et les déformations crâniennes, qui ne peuvent être, en tant que tels, attribués uniquement aux 


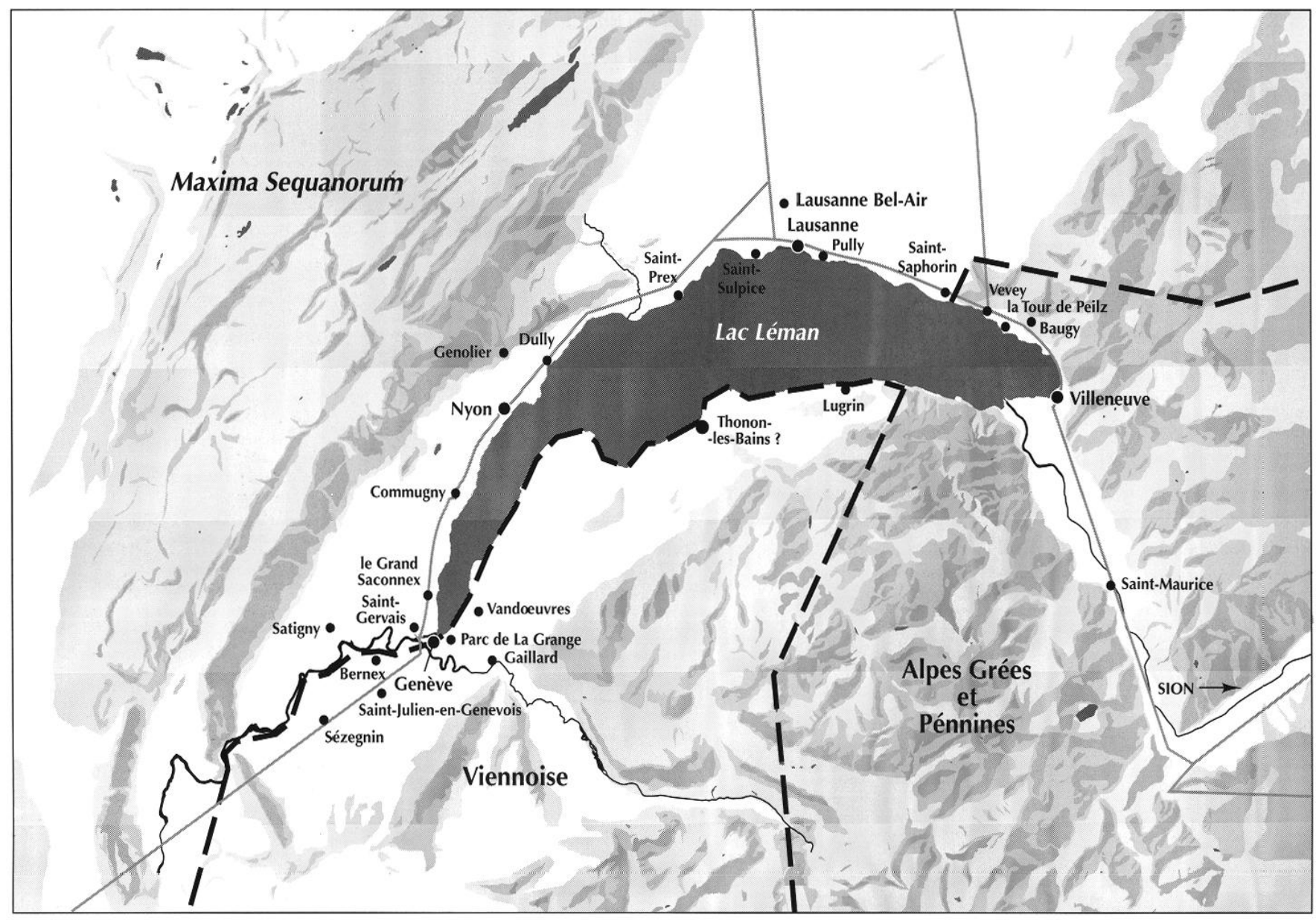

Fig. 16 - Principaux sites du Bas-Empire cités dans le texle (dessin Archéodunum, SA).

Burgondes, prouvent cependant l'installation de ce peuple en Sapaudia (Buchet, 1988; Simon, 1995). Les nécropoles qui ont livré ce type de vestiges se concentrent en effet sur la rive nord du Léman - plus particulièrement entre Genève et Lausanne - et jusqu'à Yverdon-les-Bains, au sud-ouest du lac de Neuchâtel (Marti et al., 1994, p. 28). Le nord du bassin lémanique semble donc avoir constitué le cœur du nouveau royaume.

Face à ces mutations, la permanence de l'habitat est remarquable, dans les villes importantes, mais aussi dans les campagnes. Au cours du IV c s., Genève, qui devient le siège d'un évêché, prend de plus en plus d'importance, alors que l'on observe un déclin de la colonie de Nyon (Bonnet, 1993, p. 14-21). À l'extension administrative de la nouvelle cité s'oppose le démantèlement et le remblaiement de la ville du Haut-Empire, aboutissant à un remodelage complet du périmètre urbain, qui ne couvre plus qu'une dizaine d'hectares, entre la colline de Saint-
Pierre et les berges du lac, mais fermé par une puissante enceinte, en partie constituée de blocs de pierres récupérés sur le forum de Nyon (Bonnet, 1986, p. 52-54; 1994, p. 38-39). Dans le dernier quart du III's. est édifié un vaste complexe administratif, vraisemblablement le praetorium, en partie recouvert, dès 381 par la première cathédrale et son baptistère (Bonnet, 1993, p. 14-27). L'évolution du quartier sera dès lors rythmée par l'agrandissement de l'épiscopat ; la construction d'une seconde cathédrale dans le courant du $\mathrm{V}^{\mathrm{e}} \mathrm{s}$. forme désormais un groupe cathédral. Le vicus de Lousonna-Vidy demeure partiellement occupé jusqu'à la fin du IV ${ }^{\mathrm{c}} \mathrm{s}$. au moins, tandis que la colline de la Cité révèle dès le IV $\mathrm{s}$. la présence de vastes bâtiments maçonnés, occupés jusqu'au $\mathrm{VI}^{\mathrm{c}}$ s. (Egloff, Farjon, 1983 ; Wolf, 1995). Plus à l'est, la fouille d'un secteur du vicus de Viviscus-Vevey confirme l'occupation des demeures jusqu'au début du $\mathrm{V}^{\mathrm{e}} \mathrm{s}$. et Massongex reste fréquenté jusqu'au $\mathrm{VII}^{\mathrm{e}} \mathrm{s}$. au moins. 
Si le déclin de la colonie équestre au profit de Genève ne fait guère de doute, de nombreux indices attestent que Nyon est encore occupée au Bas-Empire et dans les premiers siècles du Haut Moyen Âge. Des monnaies du IV ${ }^{e}$ s. ont été mises au jour dans la ville, et en particulier dans l'amphithéâtre (Rossi éd., 1998). Quelques pièces mérovingiennes et carolingiennes attestent une fréquentation au moins sporadique des lieux durant le Haut Moyen Âge. En outre, deux absides orientées à l'est ont été découvertes de part et d'autre du forum. L'une de ces absides, située sous le temple actuel, ne semble pas être antérieure à l'époque carolingienne ${ }^{15}$. L'autre pourrait être plus ancienne, si l'on en croit la typologie des sépultures mises au jour à proximité (Rossi, 1989, p. 259-260). Il est possible que l'habitat se soit concentré à l'intérieur des murs du forum dès la fin de l'époque romaine, mais le mobilier est presque inexisant (Rossi éd., 1998).

La trame des résidences rurales présente aussi une étonnante stabilité. Dans la région genevoise, plusieurs domaines sont agrandis et enrichis (Vandœuvre et Parc de la Grange), et on rencontre des fondations nouvelles (Sézegnin, Bursins). La mansio de Saint-Saphorin ainsi que les villae de Pully et de Montreux, en Baugy, sont également habitées en tout cas jusqu'au début du $V^{e}$ s. ${ }^{16}$. En amont du Léman, plusieurs villae sont encore utilisées en plein $\mathrm{V}^{\mathrm{c}} \mathrm{s}$., telles celles de Monthey, de Conthey, de Sion, Sous-le-Scex, et de Saint-Ginier à Sierre ${ }^{17}$. On constate par ailleurs qu'un certain nombre d'églises rurales se développent en étroite relation avec des établissements gallo-romains, comme à Satigny, Vandœuvre ou encore Saint-Saphorin (Bonnet, 1994, 1997 ; Terrier et al., 1993 ; Eggenberger, Auberson, 1992). Cette densité est confirmée par celle des nécropoles, qui sont notre principale source d'information sur

\footnotetext{
15. Les fouilles exécutées par L. Blondel en 1925-1926 n'ont malheureusement pas fait l'objet d'un compte rendu détaillé ( $c f$. Revue historique vaudoise, 35, 1927, p. 205). Plusieurs plans annotés sont néanmoins conservés dans les archives de la section des Monuments historiques et archéologiques de Lausanne, aux archives cantonales vaudoises, à Lausanne, ainsi qu'au musée historique de Nyon. Nous remercions M. Klausener et V. Lieber de nous avoir permis de consulter ces documents.
}

16. Saint-Saphorin VD : Eggenberger, Auberson, 1992 ; Pully VD : Weidmann, 1978; Montrcux, En Baugy VD : Morcl, 1991.

17. Monthey VS : Steiner, 1995 ; Conthey VS, Sion Sous-le-Scex VS : L.ehner, 1987 ; Sierre, Saint-Ginier VS : Lehner, 1993. l'occupation du territoire durant l'Antiquité tardive ${ }^{18}$. Or elles sont bien souvent proches de sites gallo-romains (Steiner, 1993, p. 13-15 et 156-163, pl. 1). Partout où elle peut être appréhendée, la densité de ces nécropoles montre que l'occupation du sol reste importante sur les deux rives du lac.

L'importance des voies lacustres et fluviales semble croître à l'époque romaine tardive, et se maintient durant le Haut Moyen Âge ${ }^{19}$. Si les sources écrites sont malheureusement muettes à propos du Léman, on dispose de quelques textes concernant la navigation sur les cours d'eau avoisinants. Un passage de la Notitia Dignitatum atteste ainsi qu'une flottille stationnait au IV $^{\text {e }}$ s. dans le castrum d'Eburodunum (Yverdon-les-Bains, VD), point de rupture de charge entre les bassins du Rhône et du Rhin (Van Berchem, 1982, p. 265-274). La découverte d'une barque en bois datée de la fin du IVe $s$. dans la même ville est un témoignage de cette navigation (fig. 14) (Arnold, 1992; Terrier, 1997). Plusieurs écrits attestent d'autre part le maintien des corps de nautonniers sur le Rhône et la Saône jusqu'au début du VIc s. ${ }^{20}$. L'archéologie n'apporte guère plus de renseignements. La fouille des ports successifs de Genève a néanmoins révélé plusieurs aménagements remontant au BasEmpire (Bonnet et al., 1989, p. 7-8 ; Broillet et al., 1997, p. 26-30). Certaines de ces constructions, notamment des tronçons d'une digue, réutilisent des blocs provenant vraisemblablement du forum de Nyon. Ces nouvelles installations portuaires sont à mettre en relation avec l'acheminement des matériaux nécessaires à la construction de l'enceinte édifiée sur la colline, au moment où Genève accède au rang de cité. La découverte de blocs extraits des monuments publics nyonnais en divers endroits de la côte lémanique, en particulier à Lausanne, Vevey et Villeneuve, permet d'ailleurs de supposer la continuité d'utilisation d'autres ports. En outre, un dépotoir décou-

18. Cf. Colardelle, 1983, p. $301-344$ et 451 , fig. 141 p. 384 ; Privati, 1983 , p. 77-85 ; Auberson, 1987 ; Kaencl, Crotti, 1993, p. 23-47 ; Stciner, 1995.

19. Nous remercions chaleurcusement Justin Favrod de nous avoir autorisés à reprendre ici des informations qui paraîtront dans L. Steiner, F. Menna, La nécropole du Pré de la Cure à Yverdon-lesBains, Cahiers d'archéologie romande, Lausanne. Sur la navigation au Haut Moyen Âge, cf. Bautier, 1989 ; Contaminc et al., 1993, p. 27-28 et 48-49.

20. Cf. P. Varin (éd.), Vie de saint Eutrope d'Orange, Bulletin du Comité historique des monuments écrits de l'Histoire de France, 1, 1849, p. 58-59; R. de Salis (éd.), Lex Romana Burgundionum. Monumenta Germaniae Historica, Leges, II/1 (1892), XLVI, p. 162-163. 
vert à proximité de la villa de Sion, Sous-le-Scex, et comportant plusieurs dizaines d'amphores provenant d'Afrique du Nord (spatheia) et de Palestine, ainsi que de la sigillée africaine et de la vaisselle en dérivées de sigillées paléochrétiennes, souligne l'intensité des échanges commerciaux entre le Valais et la Méditerranée. Or ces échanges se faisaient certainement par voie d'eau (Dubuis et al., 1987).

$$
\text { * * }
$$

On l'aura constaté, cette tentative de bilan archéologique est avant tout terrestre. De fait, l'archéologie lémanique, qui souffre d'ailleurs d'une grave lacune sur la côte française du lac, est avant tout une archéologie d'urgence urbaine. De ce fait, nous ignorons tout, par exemple, de la pêche, qui a été longtemps une des ressources de la région. On ne sait pas grand chose non plus sur l'aménagement des terrains ruraux, en termes de parcellaire, de drainage ou d'irrigation, ni, nous l'avons relevé, sur l'activité économique des villae, en particulier dans leur relation avec le lac. En un sens, cet exposé aura surtout mis en évidence des lacunes dans la recherche archéologique lémanique, et surtout l'absence de programmes communs aux entités politiques modernes concernées.

Pourtant, la relation de l'homme et du lac peut être appréhendée à travers certains éléments. D'abord l'adaptation à l'instabilité des rives et aux variations de niveau. Dès la dernière phase de l'Âge du Fer (LT D1/LT III), les populations locales ont conçu des agglomérations plus pérennes que celles des « lacustres », en relation avec un réseau routier en développement. La colline de Saint-Pierre à Genève, cœur de la ville protohistorique, gallo-romaine puis médiévale, et l'extraordinaire pérennité d'occupation d'une villa comme celle du Parc de la Grange le prouvent. Elles ont préféré des établissements un peu plus éloignés du lac, au débouché de rivières pérennes, tout en développant des aménagements particuliers, parfois très importants, sur les berges (Genève - port et villa de La Grange -, Lausanne-Vidy, et maintenant peut-être Nyon), voire sur les deux berges lorsqu'il y avait un pont à contrôler (Genève, SaintGervais). La continuité d'occupation de la fin de La Tène au Bas-Empire montre que ces choix étaient judicieux malgré une importante transgression du niveau du lac.
Ensuite, le lac apparaît comme un trait d'union entre ses riverains, qui passaient d'une province à l'autre sans difficulté, comme le montrent bien les inscriptions des Viennois établis à Nyon. Le simple fait que l'on ait pu abattre le forum de Nyon pour fortifier Genève montre qu'il y avait une étroite communauté d'intérêt entre les deux villes. L'archéologie n'apporte aucun élément pour soutenir l'idée que le lac et le Rhône aient constitué une frontière. La basilique et les aménagements du rivage de Lousonna trahissent en outre la vocation commerciale des populations locales qui savaient profiter de la position privilégiée du Léman au carrefour de nombreuses voies. L'existence d'une corporation propre aux nautes du Léman, distincts des puissants nautes du Rhône et de la Saône, ainsi que des non moins puissants negotiatores Cisalpini el Transalpini le confirme. De ce point de vue, les nouvelles données chronologiques acquises en particulier dans le canton de Genève nous interpellent. Il semble bien qu'il y ait eu un fort dynamisme routier, attesté par des ponts et par le port de Genève, vers 120 avant J.-C. (au même moment que les maisons en bois de Besançon et que la dernière réfection du pont de Cornaux à La Tène), c'est-à-dire immédiatement après la soumission des Allobroges. Ces derniers se sont-ils spontanément tournés vers le nord ? Y a-t-il eu une intervention de Rome? Nous n'en savons rien, mais il est clair que, dès ce moment, le Léman est perçu comme une plaque tournante. Cette organisation contraste avec l'imprécision des sources écrites, et suppose de la part des responsables une connaissance précise de la géographie lémanique et de ses potentialités à longue distance. Pourtant, cette unité économique, et jusqu'à un certain point culturelle, nous l'avons vu, n'a jamais justifié d'unité politique, pas même au BasEmpire, sauf peut-être au temps des Burgondes. La géographie paraît ici déterminante : si les frontières ont varié, elles ont bien souvent intégré, avec quelques variantes, la classique tripartition naturelle du lac en trois secteurs : " petit lac ", allobroge, " grand lac ", helvète, et " haut lac », valaisan, que distinguent le relief, le climat et la nature du lac. Le paysage, souvent mouvementé du bassin lémanique paraît avoir eu un impact fort sur l'implantation humaine. Le relatif fiasco de la colonie de Nyon et le choix des cités portuaires protohistoriques de Genève et Lausanne pour fixer des évêques le montrent assez. 